\title{
Noncholinergic Lesions of the Medial Septum Impair Sequential Learning of Different Spatial Locations
}

\author{
Trisha A. Dwyer, ${ }^{1,2}$ Richard J. Servatius, ${ }^{3,4}$ and Kevin C. H. Pang ${ }^{1,2,3}$ \\ IJ. P. Scott Center for Neuroscience, Mind, and Behavior and '2Department of Psychology, Bowling Green State University, Bowling Green, Ohio 43403, \\ ${ }^{3}$ NeuroBehavioral Laboratory, Department of Veterans Affairs, New Jersey Health Care System, East Orange, New Jersey 07018, and ${ }^{4}$ Department of \\ Neuroscience, New Jersey Medical School, Newark, New Jersey 07102
}

The medial septum and diagonal band of Broca (MSDB) are major afferents to the hippocampus and are important for learning, memory, and hippocampal theta rhythm. In the present study, we assessed the effect of cholinergic or noncholinergic MSDB lesions on the sequential learning of different goal locations in the same environment, a type of task that is proposed to require hippocampal theta rhythm. Rats were administered saline, 192-IgG saporin (SAP), or kainic acid (KA) into the MSDB and then behaviorally tested. On any day, a single arm of a radial maze was rewarded with food, but the location of this rewarded arm changed between days. As in previous studies, intraseptal SAP reduced the number of cholinergic neurons although sparing GABAergic septohippocampal neurons. KA had the reverse effect, reducing GABAergic septohippocampal neurons and sparing cholinergic neurons. KA, but not SAP, impaired performance on the repeated acquisition task. Saline and SAP rats showed rapid within-session learning, whereas KA rats were much slower to learn the goal location. Performance on a $30 \mathrm{~min}$ retention trial was also impaired, although this may be attributable to incomplete acquisition. These findings provide evidence that noncholinergic, but not cholinergic, MSDB neurons are important in helping the animal deal with high loads of memory interference, and provides partial support for the idea that hippocampal theta rhythm is involved.

Key words: acetylcholine; GABA; learning; memory; hippocampus; theta rhythm

\section{Introduction}

The medial septum and the vertical limb of the diagonal band of Broca (MSDB) make extensive connections with the hippocampus (Amaral and Kurz, 1985; Jakab and Leranth, 1995), are important for learning and memory (Winson, 1978; Kesner et al., 1986), and may contribute to the memory impairments in aging and Alzheimer's disease (Bartus et al., 1982; Coyle et al., 1983). Although the importance of the MSDB in spatial memory is generally accepted, the exact role of its constituent neurons in cognition is less clear. Selective damage of cholinergic MSDB neurons using 192-IgG saporin (SAP) has generated equivocal results with both impairments and no impairments reported (BergerSweeney et al., 1994; Baxter et al., 1995; Dornan et al., 1996; Walsh et al., 1996; Pang and Nocera, 1999; Chang and Gold, 2004). When deficits are observed, the impairments with selective cholinergic lesions are generally smaller than those observed with nonselective MSDB lesions, suggesting a role for noncholinergic MSDB neurons in spatial memory. The most prominent of these noncholinergic neurons is the GABAergic septohippocampal neurons, which, together with the cholinergic neurons, are

Received Sept. 25, 2006; revised Dec. 9, 2006; accepted Dec. 11, 2006

This work was supported by the National Institutes of Health, the Department of Veterans Affairs Medical Research, and Mrs. Dorothy Price. We thank Caitlin Spontelli for her assistance on this project.

Correspondence should be addressed to Dr. Kevin Pang, NeuroBehavioral Laboratory, Veterans Affairs Medical Center, 385 Tremont Avenue, Mailstop 129, East Orange, NJ 07018. E-mail: pang@njneuromed.org. DOl:10.1523/JNEUROSCI.4189-06.2007

Copyright $\odot 2007$ Society for Neuroscience $\quad$ 0270-6474/07/270299-05\$15.00/0 responsible for $\sim 80-90 \%$ of the septohippocampal pathway (Freund, 1989; Kiss et al., 1997).

Studies investigating the functional significance of noncholinergic MSDB neurons have been sparse. Small amounts of kainic acid (KA) or ibotenic acid have been used to damage noncholinergic, but not cholinergic, MSDB neurons (Malthe-Sorenssen et al., 1980; Cahill and Baxter, 2001; Pang et al., 2001; Yoder and Pang, 2005). Despite the extensive damage to GABAergic septohippocampal neurons, spatial working and reference memory were unimpaired (Pang et al., 2001). However, damage to both cholinergic and noncholinergic neurons impaired both types of memory, suggesting a functional redundancy between MSDB neurons.

Selective cholinergic and noncholinergic MSDB lesions disrupt hippocampal theta rhythm and one hypothesis of theta rhythm function is that the rhythm serves to minimize memory interference (Hasselmo, 2005). Therefore, the present study investigated the effects of cholinergic or noncholinergic MSDB damage in a task that requires sequential learning of different goal locations in the same environment, effectively enhancing proactive interference (Whishaw, 1985). If the theta rhythm serves to reduce interference, intraseptal SAP or KA should impair performance on this task.

\section{Materials and Methods \\ Subjects}

Male Long-Evans rats ( $n=30 ; 250-350 \mathrm{~g})$ were used in this study. Rats were housed one or two per cage in a colony room on a $12 \mathrm{~h}$ light/dark cycle with lights on at 7:00 A.M. Training and testing were performed 
during the light phase of the light/dark cycle. All procedures were conducted in accordance with the National Institutes of Health Guide for the Care and Use of Laboratory Animals and were approved by the Bowling Green State University Institutional Animal Care and Use Committee.

\section{Surgery}

Surgical procedures were as described previously (Pang et al., 2001). During anesthesia with sodium secobarbital or isoflurane, the skull surface was leveled and the needle of a Hamilton syringe was inserted into the MS to administer saline, KA ( 1 or $0.75 \mu \mathrm{g} / \mu \mathrm{l})$, or SAP $(0.1$ or 0.115 $\mu \mathrm{g} / \mu \mathrm{l})$. KA $(0.5 \mu \mathrm{l})$ was administered into the MS at $0.6 \mathrm{~mm}$ anterior and $1.5 \mathrm{~mm}$ lateral from bregma, $-6.6 \mathrm{~mm}$ from brain surface, and $15^{\circ}$ toward midline to target midline GABAergic septohippocampal neurons. SAP $(0.3 \mu$ linto each hemisphere) was administered at $0.6 \mathrm{~mm}$ anterior and $0.5 \mathrm{~mm}$ lateral from bregma and $-6.6 \mathrm{~mm}$ from brain surface to target the laterally located cholinergic neurons. Half of the control animals received saline at each set of coordinates. All animals received additional injections into each $\mathrm{DB}(0.4 \mu \mathrm{l}$ into each hemisphere at $+0.6 \mathrm{~mm}$ and $\pm 0.5 \mathrm{~mm}$ from bregma and $-7.8 \mathrm{~mm}$ from brain surface). Solutions were administered at a rate of $0.1 \mu \mathrm{l} / \mathrm{min}$.

\section{Apparatus}

A radial arm maze (Lafayette Instruments, Lafayette, IN) had eight black metal arms ( $27 \mathrm{~cm}$ length; $4 \mathrm{~cm}$ width) that extended from an octagonal center ( $14 \mathrm{~cm}$ diameter). Plexiglas doors surrounded the octagonal center. The maze was located $35 \mathrm{~cm}$ above the floor. At the end of each arm was a recessed food cup.

\section{Behavior}

Training. Rats were trained to explore the maze by baiting all arms with sugared cereal after food restriction (85\% ad libitum weight). Sessions lasted a maximum of $10 \mathrm{~min}$. Acclimation to the mechanical doors was performed by closing the doors after entry into an arm. During this training phase, the door remained closed for the rest of the session. After consuming the food, rats were placed back in the start arm and allowed to explore until all arms had been visited. Testing began when all maze arms were visited in $10 \mathrm{~min}$.

Testing. Testing occurred over nine daily sessions. During a test session, one arm (goal arm) was baited with a piece of sugared cereal. The location of the goal arm was the same for all trials in a test session. However, the location of the goal arm changed between sessions. The test session consisted of six trials; a trial ended when the rat made a correct entry into the goal arm or made six errors (entries into non-goal arms). For each trial, a start arm was selected randomly from the non-goal arms, excluding the two arms adjacent to the goal arm and start arms from previous trials of the session. Therefore, the first five trials of a session used different start arms. For the sixth trial (retention trial), the start arm was the same as trial 5 .

At the beginning of a trial, a rat was placed in the start arm and allowed to explore the maze. After a correct arm entry, the rat was allowed to eat the cereal and then placed in a plastic holding cage for a $30 \mathrm{~s}$ intertrial interval. During the intertrial interval, the maze was wiped with a wet cloth and the goal arm was rebaited. After an incorrect entry, the rat was removed from the arm, immediately placed in the start arm, and allowed to search for the goal arm. After six incorrect choices, the trial ended and the rat was placed in the holding cage for a $30 \mathrm{~s}$ intertrial interval. The next trial was started after the intertrial interval. A 30 min intertrial interval was used between trials 5 and 6 to assess retention of the location of the goal arm.

\section{Data analysis}

Data from trials 1-5 were analyzed separately from trial 6 . Learning across sessions was determined by computing the session mean of errors (trials 2-5) for each rat. For the analysis of within-session learning, the mean number of errors was calculated for each trial across all sessions. A similar analysis was performed for sessions blocked into early (sessions 1-3), intermediate (sessions 4-6), and late (sessions 7-9) sessions. A mixed-design ANOVA with either session or trials as a within-subject factor and drug treatment as a between-subjects factor was performed for statistical evaluation (SPSS for Windows, version 11.5; SPSS, Chicago,
IL). For trial 6, the mean number of errors was computed across all sessions and analyzed using a one-way ANOVA with drug treatment as a between-subjects factor. Post hoc analyses were performed using Tukey's honestly significant difference test. Mauchly's test was used to determine violations in the assumptions of sphericity for repeated-measure factors and Greenhouse-Geisser corrections were used in the appropriate situation to correct for violations (Geisser and Greenhouse, 1958). Corrected statistics were only reported when the uncorrected and corrected $p$ values disagreed with regard to significance.

\section{Histology}

After perfusion with $4 \%$ paraformaldehyde, brains were removed, cryoprotected in $30 \%$ sucrose, and sectioned at $50 \mu \mathrm{m}$ using a freezing microtome. Sections containing the MSDB were prepared for immunocytochemical detection of choline acetyltransferase (ChAT-ir) (1:500, AB144P; Millipore, Temecula, CA) and parvalbumin (PV-ir) (1:1000, P3088; Sigma, St. Louis, MO) as reported previously (Smith and Pang, 2005).

\section{Stereology}

Estimates of the number of ChAT-ir and PV-ir MSDB neurons for all groups were obtained using standard stereology procedures (West, 1999). Counts were performed by a person who was blind to the treatments. Every third section of the entire MSDB was counted, including the MS, vertical limb of the DB, and horizontal limb of the DB. Three rats from each condition were randomly selected for counting from the rats used in the behavioral analysis. Stereology was performed using the optical fractionator method (Stereo Investigator version 7.0; MicroBrightField, Colchester, VT) on a microscope with an $x$-, $y$-, z-axis motorized stage (Bio Point 30; Ludl Electronic Products, Hawthorne, NY). Leading edges of ChAT-ir or PV-ir cell bodies were counted using a $40 \times$ objective lens (NeoFluar, 0.75 NA; Zeiss, Oberkochen, Germany). Cells in the uppermost focal plane $(2 \mu \mathrm{m})$ were not counted. The counting frame had a height of $15 \mu \mathrm{m}$ and was $150 \mu \mathrm{m} \times 100 \mu \mathrm{m}$ in size.

\section{Results \\ Histology}

The effects of intraseptal saline, SAP, and KA on cholinergic and GABAergic septohippocampal neurons were assessed by counting the number of ChAT-ir and PV-ir MSDB neurons, respectively. SAP reduced ChAT-ir neurons by $63.2 \%$ (Figs. 1, 2). In contrast, PV-ir neurons were reduced by only $21.7 \%$. Intraseptal KA produced a cell loss pattern that was opposite that by SAP (Figs. 1, 2). KA reduced PV-ir neurons by $75.5 \%$ and decreased cholinergic neurons by only $21.1 \%$. Thus, intraseptal SAP and KA produced complementary patterns of damage on cholinergic and GABAergic septohippocampal neurons.

\section{Repeated acquisition task}

Intraseptal KA impaired performance on the repeated acquisition task, affecting both between- and within-session learning. KA animals were impaired across all test sessions (Fig. $3 A$ ). All groups learned the task, as demonstrated by a gradual reduction in errors across sessions $\left(F_{(8,216)}=14.04 ; p<0.01\right)$. Overall, treatment impaired performance (main effect of treatment, $\left.F_{(2,27)}=6.41 ; p<0.01\right)$, but sessions and treatment did not interact $\left(F_{(16,216)}=0.76\right)$. Post hoc analysis revealed the KA group made more errors than the saline $(p<0.01)$ and $\operatorname{SAP}(p=0.01)$, but SAP and saline groups did not differ.

Within-session learning was compromised by intraseptal KA (Fig. 3B). Learning occurred within sessions (main effect of trials, $\left.F_{(4,108)}=41.26 ; p<0.01\right)$ and treatment impaired performance (main effect of treatment, $F_{(2,27)}=4.44 ; p=0.02$ ). Furthermore, trials and treatment interacted $\left(F_{(8,108)}=2.28 ; p=0.03\right)$. However, the Greenhouse-Geisser correction made the interaction marginally significant $(p=0.06)$. Post hoc analysis showed that 


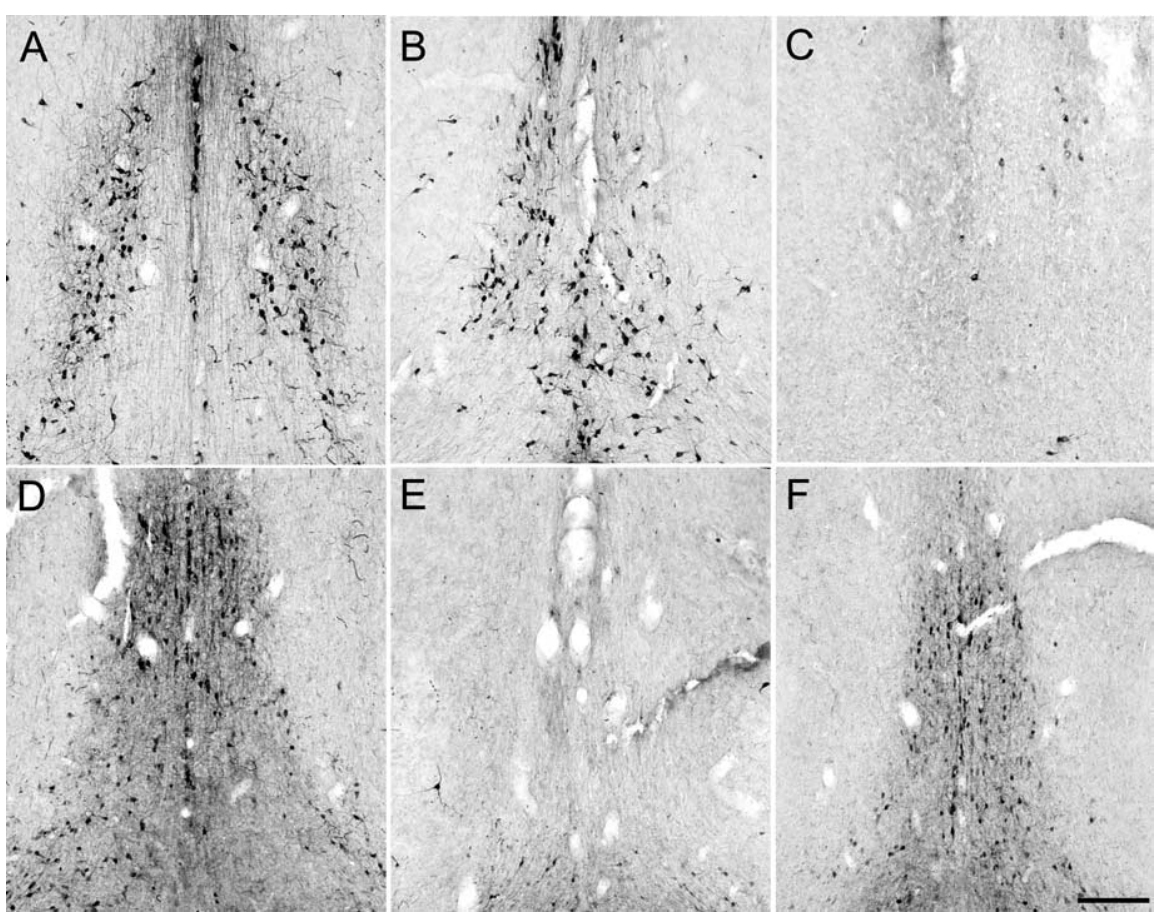

Figure 1. $\quad A-F$, Photomicrographs of the MSDB after intraseptal administration of saline $(A, D), K A(B, E)$, or SAP $(C, F)$. KA and SAP had complementary effects on cholinergic $(\boldsymbol{A}, \boldsymbol{B}, \boldsymbol{C})$ and GABAergic $(\boldsymbol{D}, \boldsymbol{E}, \boldsymbol{F})$ septohippocampal neurons. Scale bar, $200 \mu \mathrm{m}$.

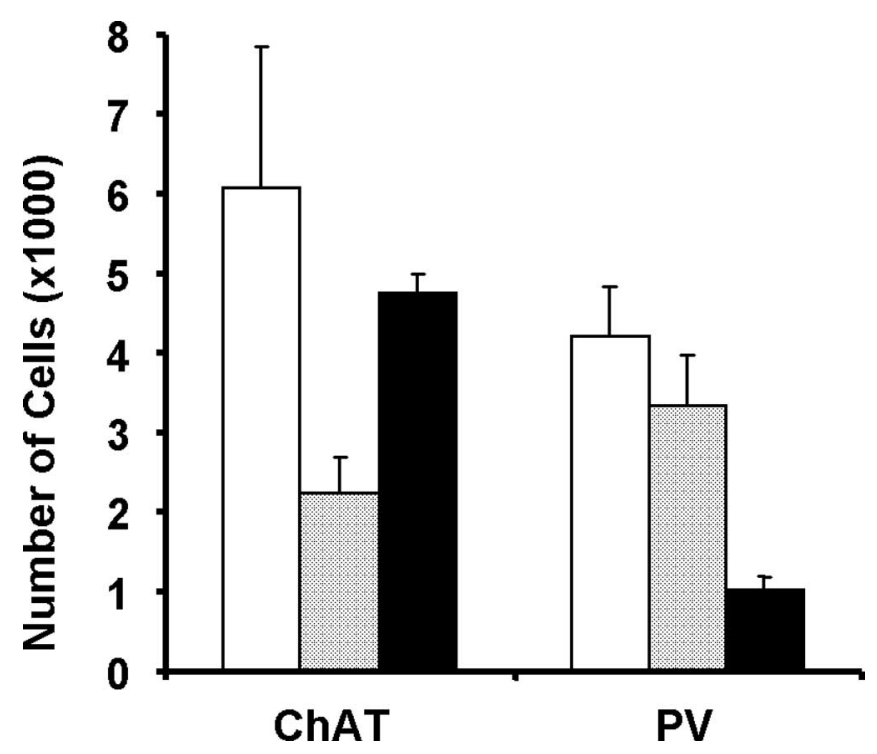

Figure 2. Counts of cholinergic (ChAT) and GABAergic (PV) septohippocampal neurons after intraseptal saline (open), SAP (gray), or KA (black) administration.

$\mathrm{KA}$, but not SAP rats made more errors than saline animals $(p=$ $0.02)$. Differences between SAP and KA groups were marginally significant $(p=0.08)$.

Within-session learning was different across test session blocks. MSDB treatment only affected learning in the intermediate, but not early or late session blocks (main effect of treatment, $F_{(2,27)}=5.25 ; p=0.01$ ) (data not shown). However, the pattern of results for all blocks was similar to the overall session results (Fig. 3B). Post hoc analysis of the Intermediate test block revealed that KA rats made more errors than saline animals $(p<0.01)$ and SAP animals did not differ from either group.

Error analysis was performed on entries into the goal arm of the previous day ("previous goal arm") and entries into arms previously entered in the current session ("repeat entries"). All rats entered the previous goal arm more on trial 1 than on subsequent trials (main effect of trials, $\left.F_{(2,108)}=51.85 ; p<0.01\right)$ (Fig. 3C). However, treatments did not alter this pattern (main effect of treatment, $\left.F_{(2,27)}=0.92\right)$. The interaction of treatment and trials was marginally significant $\left(F_{(8,108)}=2.02 ; p=0.051\right)$.

Repeat entries for saline and SAP groups were similar to each other and were unchanged across trials (Fig. 3D). In contrast, the KA group increased repeat entries between trials 1 and 2 and then remained at this elevated level for subsequent trials (main effect of trials $F_{(4,108)}=4.06, p<0.01$; main effect of treatment, $F_{(2,27)}=4.57, p=0.02$; trials by treatment interaction, $F_{(8,108)}=3.56, p<$ 0.01). Post hoc analysis demonstrated a clear difference between KA and saline ( $p=0.02)$ groups and the difference between KA and SAP was marginally significant $(p=0.07)$. Saline and SAP groups did not differ.

$\mathrm{KA}$ rats made more errors on a $30 \mathrm{~min}$ retention test $\left(F_{(2,27)}=3.67 ; p=0.04\right)$ (trial 6) (Fig. 3E). Post hoc analysis demonstrated marginally significant differences between KA rats and both saline $(p=0.06)$ and SAP $(p=0.08)$ groups. SAP and saline rats did not differ. Analyses by session block showed that treatment impacted memory retention during the intermediate $\left(F_{(2,27)}=3.31 ; p=0.05\right)$ and late $\left(F_{(2,27)}=3.53\right.$; $p=0.04)$ sessions, but not during the early session $\left(F_{(2,27)}=\right.$ 1.33) (data not shown). Post hoc analysis revealed differences between KA and saline rats $(p=0.05)$ in the intermediate block, with marginally significant differences between KA and both saline $(p=0.07)$ and SAP $(p=0.07)$ groups in the late sessions. Saline and SAP groups did not differ during any of the session blocks.

\section{Discussion}

The present study assessed the roles of different populations of MSDB neurons in a repeated acquisition task. Noncholinergic MSDB lesions using KA were severely impaired in learning the location of a goal that changed every day. In contrast, rats with selective damage of cholinergic MSDB neurons were unimpaired. The nature of the deficit suggests that noncholinergic neurons may be important in controlling proactive interference.

Damage to MSDB neurons was produced by intraseptal administration of SAP and KA. As in previous studies, SAP administration severely damaged cholinergic (ChAT-ir) neurons with a small reduction in GABAergic septohippocampal (PV-ir) neurons. In contrast, KA preferentially damaged GABAergic septohippocampal neurons, while sparing most of the cholinergic MSDB neurons. KA may also damage other noncholinergic neurons in the MSDB, and we refer to the damage produced by intraseptal KA as noncholinergic because of this uncertainty. However, some GABAergic MSDB neurons are spared using doses of KA similar to those in the present study, so not all noncholinergic neurons are damaged equivalently (Pang et al., 2001).

Over the course of the study, KA rats consistently had more 
errors than saline rats. Performance of SAP rats was not different from the saline group. More importantly, KA animals were impaired in their within-session learning. On trial 1, all groups made a similar number of errors, as expected when the goal arm location changes daily. However, saline and SAP animals quickly learned the location of the goal arm, as demonstrated by a rapid reduction in the number of errors on subsequent trials. In contrast, KA animals were slower to learn the goal location and never reached the same level of performance as saline or SAP rats. KA animals were also impaired on a 30 min retention trial, probably because of incomplete learning of the goal location during the first 5 trials.

These results stand in contrast to the lack of impairments observed after intraseptal KA using standard water maze and radial maze procedures (Pang et al., 2001). The unimpaired performance suggests that noncholinergic septohippocampal neurons are not critically important for acquisition of spatial memory. One difference between the standard water maze procedure and the procedure used in the present study is that the standard water maze procedure uses an escape platform that does not change location, whereas the present study moved the goal arm daily. Thus, interference from goal locations of previous sessions is greatly increased in the present study, and KA rats may be susceptible to this proactive interference. Our error analysis, however, suggests otherwise. All animals were predisposed to enter the goal arm of the previous day on trial 1, and then rapidly learned to avoid the previous goal arm on subsequent trials. Neither KA nor SAP groups were different from the saline group in this respect. The similar performance on trial 1 also demonstrates that KA rats remembered the location of the previous day's goal as well as saline and SAP rats.

Where KA animals differed from the other groups was in the number of arms repeated in the same session. All animals had similar numbers of repeat entries on trial 1 . However, KA rats compared with saline and SAP rats had many more repeat errors on subsequent trials. Rather than interference from the goal location of the previous day, KA rats seem to have difficulty distinguishing between incorrect and correct entries within a session. This pattern of errors suggests that KA rats have more withinsession proactive interference than between-session interference.

Reduction of proactive interference has been suggested to be an important function of hippocampal theta rhythm and acetylcholine (Hasselmo et al., 1996, 2005). Hippocampal theta rhythm requires a functionally intact $\mathrm{MSDB}$, with both cholinergic and noncholinergic neurons being important (Stewart and Fox, 1990; Lee et al., 1994; Bassant et al., 1998; Yoder and Pang, 2005; Pascale Simon et al., 2006). Damage produced by intraseptal KA eliminates hippocampal theta rhythm observed in urethane anesthesia and dramatically reduces the amplitude of the rhythm during locomotion (Yoder and Pang, 2005). Therefore, the results of the present study support the idea that noncholinergic MSDB neurons reduce memory interference via hippocampal theta rhythm.

Rats that were administered intraseptal SAP were not impaired on the repeated acquisition task. This finding is consistent
B

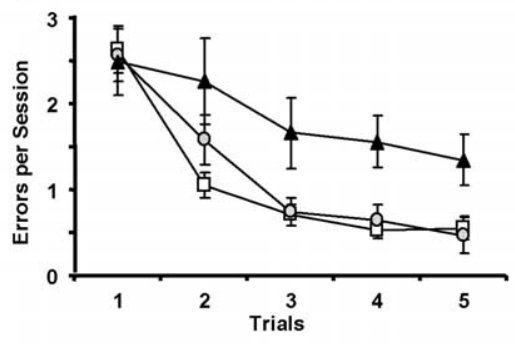

E

D
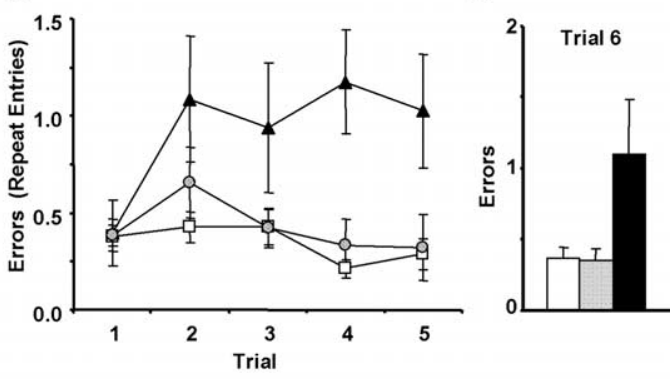$$
\text { (1) }
$$

rial

$\square$ Saline

- SAP

$\Delta \mathrm{KA}$
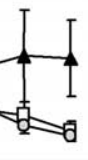

Days

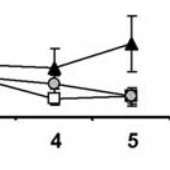

, B

Performance on a repeated acquisition task after intraseptal administration of saline, SAP, or KA. A, B, Betweenwere not different between treatments $(\boldsymbol{C}$ ), but repeat entries in the same session were increased in KA rats $(\boldsymbol{D})$. $\boldsymbol{E}$, KA also retention test.

with a number of studies that have reported no or small impairment in various types of spatial memory tasks (Berger-Sweeney et al., 1994; Baxter et al., 1995; Dornan et al., 1996; Pang and Nocera, 1999). Despite the small or lack of memory impairment after intraseptal SAP, hippocampal theta rhythm is still reduced weeks after the SAP lesion (Lee et al., 1994; Bassant et al., 1998; Yoder and Pang, 2005). The effect of intraseptal SAP on hippocampal theta rhythm resembles that produced by intraseptal KA (Yoder and Pang, 2005). If hippocampal theta rhythm is important for performance on the repeated acquisition task as suggested by the results of KA rats, it is unclear why SAP rats are unimpaired. One possibility that might help account for the difference is the lesion magnitude. KA treatment reduced GABAergic septohippocampal neurons by $76 \%$, whereas SAP eliminated $63 \%$ of the cholinergic neurons. The lack of impairment observed in the present study could be attributable to insufficient damage to cholinergic neurons. However, other studies with similar amounts or less damage (50-68\% reduction) have observed impairments using other spatial memory tasks [68\% (Shen, 1996); 50\% (Dornan et al., 1996); 62\% (Walsh et al., 1996)]. Still, it will be important for future studies to determine whether more extensive lesions of cholinergic MSDB neurons without further compromise of GABAergic MSDB neurons impair performance in this repeated acquisition task.

Another possibility is that recovery of function after the lesions may be different for cholinergic and GABAergic neurons. Recovery from damage will be dependent on a number of factors including cell type, nature of damage, and extent of damage. It is possible that cholinergic neurons have more capacity for sprouting or other compensatory mechanisms compared to GABAergic septohippocampal neurons. Despite extensive loss of some cholinergic markers after intraseptal SAP, other markers can demonstrate recovery or are spared (Chang and Gold, 2004). It remains to be seen if similar types of sparing or compensatory changes are observed in GABAergic neurons after intraseptal KA administration. 
In summary, damage to noncholinergic MSDB neurons impaired learning of different goal locations in the same environment. Lesions of the cholinergic septohippocampal neurons did not impair performance on the same task. Although the extent of the damage produced by intraseptal KA on noncholinergic MSDB neurons is unknown, it is clear that GABAergic septohippocampal neurons are severely compromised. Therefore, the present study demonstrates a dissociation between the two major components of the septohippocampal pathway. These results, together with those from previous studies, suggest that noncholinergic MSDB neurons are important for reducing proactive interference, possibly through its influence on hippocampal theta rhythm.

\section{References}

Amaral DG, Kurz J (1985) An analysis of the origins of the cholinergic and noncholinergic septal projections to the hippocampal-formation of the rat. J Comp Neurol 240:37-59.

Bartus RT, Dean RL, Beer B, Lippa AS (1982) The cholinergic hypothesis of geriatric memory dysfunction. Science 217:408-416.

Bassant MH, Jouvenceau A, Apartis E, Poindessous-Jazat F, Dutar P, Billard JM (1998) Immunolesion of the cholinergic basal forebrain: effects on functional properties of hippocampal and septal neurons. Int J Dev Neurosci 16:613-632.

Baxter MG, Bucci DJ, Gorman LK, Wiley RG, Gallagher M (1995) Selective immunotoxic lesions of basal forebrain cholinergic cells: effects on learning and memory in rats. Behav Neurosci 109:714-722.

Berger-Sweeney J, Heckers S, Mesulam MM, Wiley RG, Lappi DA, Sharma M (1994) Differential effects on spatial navigation of immunotoxininduced cholinergic lesions of the medial septal area and nucleus basalis magnocellularis. J Neurosci 14:4507-4519.

Cahill JF, Baxter MG (2001) Cholinergic and noncholinergic septal neurons modulate strategy selection in spatial learning. Eur J Neurosci 14:1856-1864.

Chang Q, Gold PE (2004) Impaired and spared cholinergic functions in the hippocampus after lesions of the medial septum/vertical limb of the diagonal band with 192 IgG-saporin. Hippocampus 14:170-179.

Coyle JT, Price DL, DeLong MR (1983) Alzheimer's disease: a disorder of cortical cholinergic innervation. Science 219:1184-1190.

Dornan WA, McCampbell AR, Tinkler GP, Hickman LJ, Bannon AW, Decker MW, Gunther KL (1996) Comparison of site-specific injections into the basal forebrain on water maze and radial arm maze performance in the male rat after immunolesioning with 192 IgG saporin. Behav Brain Res 82:93-101.

Freund TF (1989) GABAergic septohippocampal neurons contain parvalbumin. Brain Res 478:375-381.

Geisser S, Greenhouse SW (1958) An extension of Box's results on the use of the F distribution in multivariate analysis. Ann Math Stat 29:885-891.

Hasselmo ME (2005) What is the function of hippocampal theta rhythm?
Linking behavioral data to phasic properties of field potential and unit recording data. Hippocampus 15:936-949.

Hasselmo ME, Wyble BP, Wallenstein GV (1996) Encoding and retrieval of episodic memories: Role of cholinergic and GABAergic modulation in the hippocampus. Hippocampus 6:693-708.

Jakab R, Leranth C (1995) Septum. In: The rat nervous system, Ed 2 (Paxinos G, ed), pp 405-442. San Diego: Academic.

Kesner RP, Crutcher KA, Measom MO (1986) Medial septal and nucleus basalis magnocellularis lesions produce order memory deficit in rats which mimic symptomology of Alzheimer's disease. Neurobiol Aging 7:287-295.

Kiss J, Magloczky Z, Somogyi J, Freund TF (1997) Distribution of calretinin-containing neurons relative to other neurochemically identified cell types in the medial septum of the rat. Neuroscience 78:399-410.

Lee MG, Chrobak JJ, Sik A, Wiley RG, Buzsaki G (1994) Hippocampal theta activity following selective lesion of the septal cholinergic system. Neuroscience 62:1033-1047.

Malthe-Sorenssen D, Odden E, Walaas I (1980) Selective destruction by kainic acid of neurons innervated by putative glutamatergic afferents in septum and nucleus of the diagonal band. Brain Res 182:461-465.

Pang KC, Nocera R (1999) Interactions between 192-IgG and intraseptal cholinergic and GABAergic drugs: role of cholinergic medial septal neurons in spatial working memory. Behav Neurosci 113:265-275.

Pang KC, Nocera R, Secor AJ, Yoder RM (2001) GABAergic septohippocampal neurons are not necessary for spatial memory. Hippocampus 11:814-827.

Pascale Simon A, Poindessous-Jasat F, Dutar P, Epelbaum J, Bassant M (2006) Firing properties of anatomically identified neurons in the medial septum of anesthetized and unanesthetized restrained rats. J Neurosci 26:9038-9046.

Shen J, Barnes CA, Wenk GL, McNaughton BL (1996) Differential effects of selective immunotoxic lesions of medial septal cholinergic cells on spatial working and reference memory. Behav Neurosci 110:1181-1186.

Smith HR, Pang KC (2005) Orexin-saporin lesions of the medial septum impair spatial memory. Neuroscience 132:261-271.

Stewart M, Fox SE (1990) Do septal neurons pace the hippocampal theta rhythm? Trends Neurosci 13:163-168.

Walsh TJ, Herzog CD, Gandhi C, Stackman RW, Wiley RG (1996) Injection of IgG 192-saporin into the medial septum produces cholinergic hypofunction and dose-dependent working memory deficits. Brain Res 726:69-79.

West M (1999) Stereological methods for estimating the total number of neurons and synapses: issues of precision and bias. Trends Neurosci 22:51-61.

Whishaw IQ (1985) Formation of a place learning-set by the rat: a new paradigm for neuro-behavioral studies. Physiol Behav 35:139-143.

Winson J (1978) Loss of hippocampal theta rhythm results in spatial memory deficit in the rat. Science 201:160-163.

Yoder RM, Pang KC (2005) Involvement of GABAergic and cholinergic medial septal neurons in hippocampal theta rhythm. Hippocampus 15:381392. 